\title{
Effect of particle shape and fragmentation on the response of particle dampers
}

\author{
Martín Sánchez $^{* 1}$, C. Manuel Carlevaro ${ }^{\dagger 2,3}$ and Luis A. Pugnaloni ${ }^{\ddagger 1}$ \\ ${ }^{1}$ Departamento de Ingeniería Mecánica, Facultad Regional La Plata, Universidad Tecnológica Nacional, 60 esq. \\ 124 S/N, 1900 La Plata, Argentina. \\ ${ }^{2}$ Instituto de Física de Líquidos y Sistemas Biológicos (CONICET La Plata, UNLP), Calle 59 Nro 789 , 1900 La \\ Plata, Argentina. \\ ${ }^{3}$ U.D.B. Física, Facultad Regional Buenos Aires, Universidad Tecnológica Nacional, Mozart 2300, C1407IVT \\ Buenos Aires, Argentina.
}

\begin{abstract}
A particle damper (PD) is a device that can attenuate mechanical vibrations thanks to the dissipative collisions between grains contained in a cavity attached to the vibrating structure. It has been recently suggested that, under working conditions in which the damping is optimal, the PD has a universal response in the sense that the specific dissipative properties of the grains cease to be important for the design of the device. We present evidence from simulations of PDs containing grains of different size, shape and restitution coefficient, that the universal response is also valid when fragmentation of the grains occurs (generally due to intensive operation of the PD). In contrast, the welding of grains (caused by operation under high temperatures) can take the PD out of the universal response and deteriorate the attenuation. Interestingly, we observed that even at working conditions off the optimal damping, the shape of the grains remains unimportant for the response of the PD.
\end{abstract}

\section{Introduction}

A particle damper (PD) consists of a cavity partially filled with grains. This device can attenuate mechanical vibrations through friction and inelastic collisions of the grains when it is attached to a

\footnotetext{
*sanchez.martin@frlp.utn.edu.ar (M. Sánchez)

†manuel@iflysib.unlp.edu.ar (C. M. Carlevaro)

${ }^{\ddagger}$ luis.pugnaloni@frlp.utn.edu.ar (L. A. Pugnaloni)
}

vibrating structure. In recent years, PDs have been widely studied due to the good performance they have as passive vibration control system in harsh environments.

Different industries have applied this technology to control undesirable vibrations and noises. While the aerospace industry has been the pioneer in the area (Ehrgott et al., 2009; Panossian, 2002), a number of studies have been recently published with applications in the automotive (Xia et al., 2011), energy (Velichkovich and Velichkovich, 2001) and medical (Heckel et al., 2012) industries.

PDs are efficient in a wide range of frequencies (Panossian, 1992), but due to their highly nonlinear behavior (Sánchez and Carlevaro, 2012), its analysis and design present complications. PDs are the successors of impact dampers (see e.g. (Duncan et al., 2005; Grubin, 1956; Masri, 1970)), where the single body inside the cavity of these is simply replaced with a sample of granular material (Araki et al., 1985). Many studies have focused on the prediction of the main characteristics of PDs through simplified models of a single particle (Duncan et al., 2005; Friend and Kinra, 2000; Ramachandran and Lesieutre, 2008). However, the complex cooperative dynamics of the grains is ignored in these works.

The performance of a PD depends on many factors such as shape and size of the cavity, number of particles, coefficient of friction and restitution, type of excitation and operation frequencies, among many others (Marhadi and Kinra, 2005). Depend- 


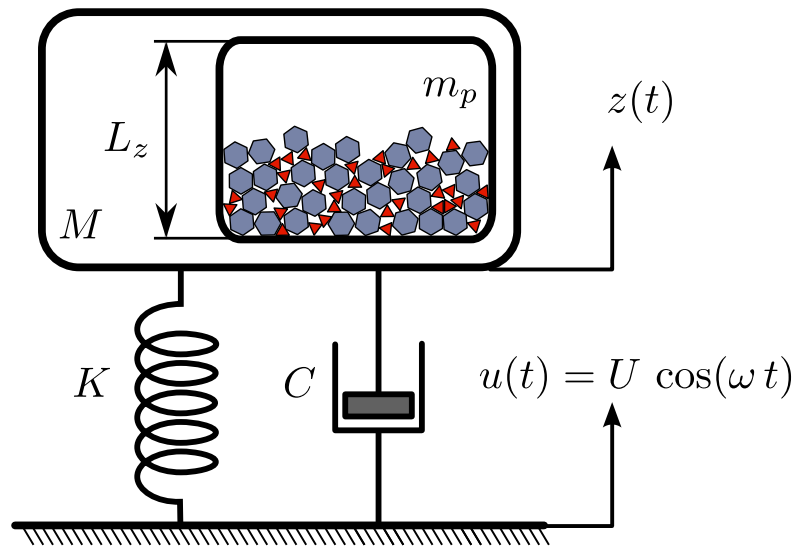

Figure 1: Schematic representation of the SDoF system with a particle damper. $M$ : mass of the primary system. $K$ : Spring constant. $C$ : structural damping. $m_{\mathrm{p}}$ : total mass of the grains. $L_{z}$ : height of the cavity. $u(t)$ : displacement imposed to the base. $z(t)$ : displacement of the primary system.

ing on some of these factors, one of the mechanism of dissipation (friction or inelastic normal collision) will dominate (Bai et al., 2009; Chen et al., 2001).

Several works have shown, that under harmonic excitation, a prismatic PD has the best performance of damping for a given value of the enclosure height $\left(L_{z}\right)$ (Papalou and Masri, 1998; Saeki, 2002). This optimal damping occurs when, near resonance, the grains impact against the floor and ceiling of the cavity in anti-phase, where the relative velocity between the granular bed and the enclosure is maximized at the time of collision $(\mathrm{Lu}$ et al., 2010; Sánchez and Pugnaloni, 2011). For an optimal PD, it has been shown that the natural frequency of the system equals the natural frequency of the undamped system (Sánchez and Pugnaloni, 2011).

Previous investigations have considered the relevance of the material of the grains (dissipative mechanisms) in PDs (Bai et al., 2009; Chen et al., 2001; Marhadi and Kinra, 2005). Recently, it has been shown that the performance of a PD is independent of the the material properties of the grains if the optimal $L_{z}$ is used (Sánchez et al., 2012). This universal behavior can be explained through the effective inelastic collapse of dense granular materials (Sánchez et al., 2012).

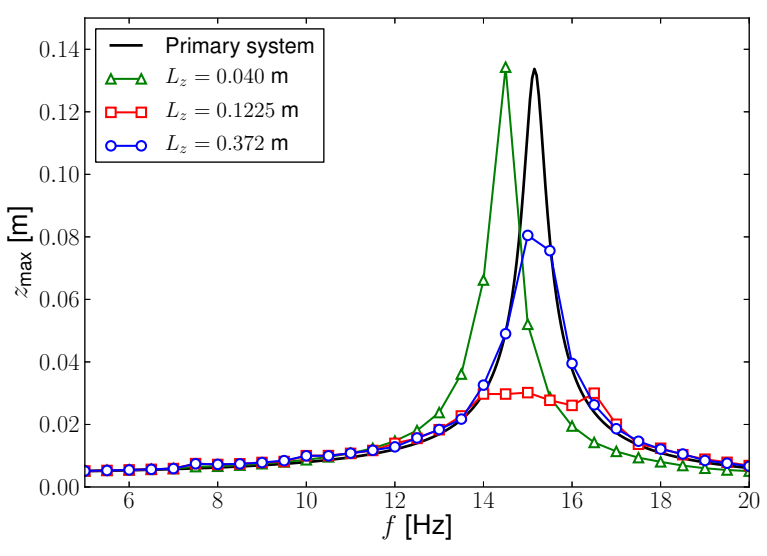

Figure 2: Frequency Response Function (FRF) for a system with $N=180$ hexagonal particles (circumscribed radius $r=0.0015 \mathrm{~m}, \mu=0.50$, and $e=0.50)$ and three different heights of the enclosure $L_{z}=0.040$ (green triangles), 0.1225 (red squares) and $0.372 \mathrm{~m}$ (blue circles). The black solid line corresponds to the response of the system without the PD. The amplitude $z_{\max }$ of the oscillation of the primary mass is platted against the excitation frequency $f$.

In this work, we show that this universality of the response of a PD remains valid even if grains of different shape are considered. We have carried out simulations with triangular, square and hexagonal grains and with different dissipative properties. Furthermore, we show that the fragmentation of particles, which is likely when operating in harsh environments, does not lead to changes in the response of the system. This phenomenon explains the low maintenance requirements of these devices, since a degradation of the granular material does not lead to any significant change of the PD performance. We also consider the possibility of welding of grains (due to operation in high temperature conditions). In this case, the vibration attenuation is observed to decrease. Interestingly, our results show that even for non-optimal PD, the shape of the grains is a factor that does not influence the response. 


\section{Simulation}

We carry out molecular dynamic type simulations (also known as discrete element method) by solving the Newton-Euler equations of motion for rigid bodies confined on a rectangular box. All simulations were done in two-dimensions (2D). Figure 1 shows a schematic representation of the system. The primary system consists of a mass $M=2.37$ $\mathrm{kg}$, a linear spring with spring constant $K=21500$ $\mathrm{Nm}^{-1}$ and a viscous damper, which accounts for any structural damping, with damping constant $C=7.6 \mathrm{Nsm}^{-1}$. Under these conditions the undamped natural frequency is $f_{0}=15.16 \mathrm{~Hz}$.

The cavity of the PD has been modeled as a rectangular box of sides $L_{x}=0.036 \mathrm{~m}$ and 0.040 $\mathrm{m}<L_{z}<0.372 \mathrm{~m}$. The walls were modeled with coefficient of friction $\mu=0.50$ and coefficient of restitution $e=0.50$. The bodies (particles) are placed in the rectangular box embedded in the primary system. The particles may have different shapes: triangles, squares and hexagons. The total mass of the particles is $m_{\mathrm{p}}=0.227 \mathrm{~kg}$. In all cases, the mass ratio $m_{\mathrm{p}} / M \approx 10 \%$ is kept constant. The restitution coefficient $(e)$ and the friction coefficient $(\mu)$ are both set to 0.50 in most simulations, but different values have been tested in the some cases.

The system is excited via a harmonic displacement $[u(t)=U \cos (\omega t)$ with $U=0.0045 \mathrm{~m}]$ of the base to which the spring and viscous damper are attached (see Fig. 1). The excitation frequency $\omega=2 \pi f$ with $f$ between $5.0 \mathrm{~Hz}$ and $20.0 \mathrm{~Hz}$.

We have considered the gravitational field $g=$ $9.8 \mathrm{~ms}^{-2}$ in the negative vertical direction. Although the primary system can only move in the $z$-direction, the grains can move freely inside the rectangular box. Particles, initially placed at random without overlaps in the box, are allowed to settle until they come to rest in order to prepare the initial packing. Then, the same protocol is applied to each sample for every frequency. For all analyzes, we used only the last $10 \%$ of the simulation time in order to ensure a stationary regime. The total simulation time corresponds to $200 \mathrm{~s}$.

The simulations were implemented by means of the Box2D library (Catto, 2012). Box2D is an open source code written in $\mathrm{C}++$ that uses a constraint solver to handle hard bodies. The equations of motion are integrated by Box2D through a symplectic Euler algorithm. At each time step of the simulation, a series of iterations (typically 20) are used to resolve penetrations between bodies (grains) through a Lagrange multiplier scheme (Catto, 2005). The contact of each polygonal particle is defined by a manifold. After resolving penetrations, the friction (through Coulomb criterion) and the inelastic collision at each contact is solved and new linear and angular velocities are assigned to each particle. The time step used to integrate the equations of motion is $0.005 \mathrm{~s}$. Box $2 \mathrm{D}$ has been previously used for simulations of granular materials subjected to mechanical vibrations and results showed remarkable agreement with experiments and other simulation approaches (Carlevaro and Pugnaloni, 2011).

In practice, Box $2 \mathrm{D}$ attains combined features of traditional discrete element methods and event driven simulations of hard particles. As it is done in event driven simulations, the impulses calculated on oblique impacts of given restitution coefficient and friction are used to update positions(orientations) and velocities(angular velocities). This effectively models hard bodies; however, instead of calculating only simple two-body collisions, the algorithm is able to calculate the effect of all neighbors on a given body simultaneously. This scheme allows for a faster and flexible modeling of non-spherical objects. However, as in event driven simulations, the force law between contacts is not defined and we lack flexibility of using sophisticated interaction models such as the Hertz-Kuwabara-Kono model. Only the effective friction and restitution of a material can be set.

\section{Results}

Figure 2 shows the Frequency Response Function (FRF) for the primary system with a PD containing $N=180$ hexagonal particles (with circumscribed radius $r=0.0015 \mathrm{~m}, \mu=0.50$, and $e=0.50$ ) for three different heights $L_{z}=0.040,0.1225$ and 0.372 $\mathrm{m}$ of the cavity. In this case, the height of the granular bed at rest is approximately $L_{0}=0.039 \mathrm{~m}$. As we can see, the results of these $2 \mathrm{D}$ simulations using hexagonal grains are consistent with previous 3D simulations (Fang and Tang, 2006; Saeki, 2002) and 


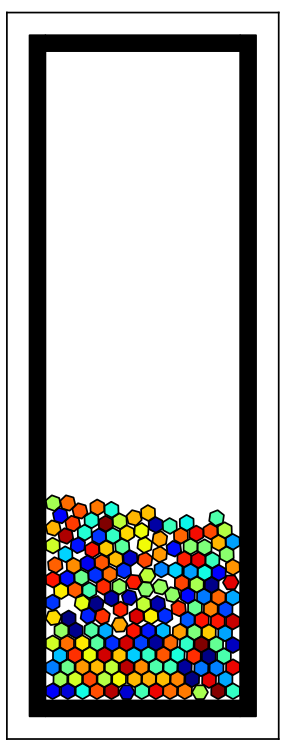

(a) Frag. 0\%

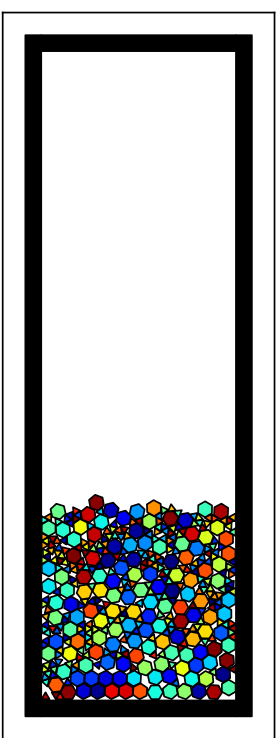

(b) Frag. $25 \%$

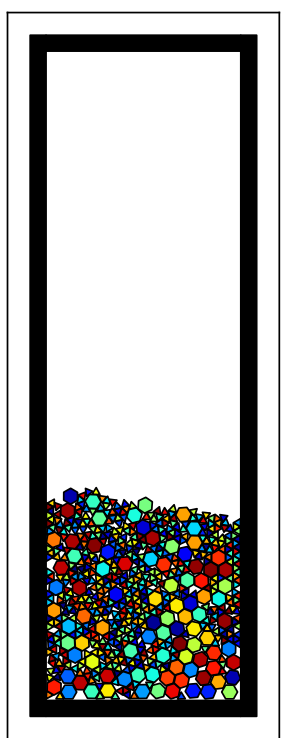

(c) Frag. $50 \%$

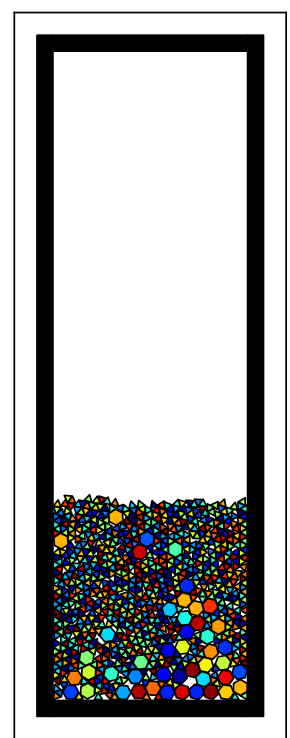

(d) Frag. $75 \%$

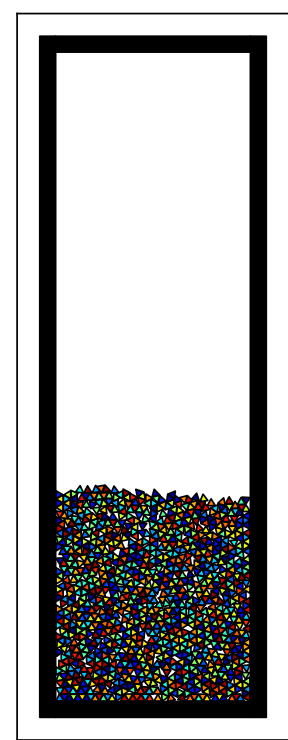

(e) Frag. $100 \%$

Figure 3: Snapshots of granular samples at rest. (a) 180 hexagons (0\% fragmentation). (b) 135 hexagons and 270 triangles (25\% fragmentation). (c) 90 hexagons and 540 triangles (50\% fragmentation). (d) 45 hexagons and 810 triangles ( $75 \%$ fragmentation). (e) 1080 Triangles (100\% fragmentation).

experiments (Liu et al., 2005; Saeki, 2002). We can observe a shift in the natural frequency of the system compatible with an effective mass varying from $M_{\text {eff }}=M$ (when $L_{z}$ is large) to $M_{\text {eff }}=M+m_{\mathrm{p}}$ (when $L_{z}$ is small enough to prevent the motion of the particles inside the cavity). Notice however that a detailed study has shown that this frequency shift does not occur in a monotonous way: The effective mass present overshoots and undershoots beyond the two limit cases ( $M$ and $\left.M+m_{\mathrm{p}}\right)$ (Sánchez and Pugnaloni, 2011).

Previous works (Papalou and Masri, 1998; Saeki, 2002; Sánchez and Pugnaloni, 2010) have shown the existence of an optimum height of the cavity of the PDs for which the best damping performance is obtained. This is also observed in our $2 \mathrm{D}$ simulations. From Fig. 2, it is clear that between the two limit cases $\left(L_{z}\right.$ small and very large) there is a height $\left(L_{z}=0.1225 \mathrm{~m}\right)$ that yields the best attenuation of the response.

Even though our simulations are twodimensional, the results obtained are consistent with the phenomenology observed in realistic particle dampers. In the next sections we will also show that $2 \mathrm{D}$ setups have the same response functions as realistic PDs under some working conditions.

\subsection{Effect of particle fragmentation}

During the operation of a PD, grains inside the cavity are prone to fragmentation and wear. This may compromise, in principle, the damping performance. To evaluate the effect of fragmentation, we carried out simulations in which hexagonal particles are progressively replaced by triangles, mimicking the fragmentation of the hexagons. A regular hexagon can be built up out of six equilateral triangles. Thus, the fragmentation of a hexagon is simulated via substitution by six identical particles which occupy the same space and have the same total mass as the original hexagon.

We have simulated various combinations of hexagons and triangles representing different degrees of fragmentation. The materials properties of the grains are shown in Table 1. The optimum height of the enclosure is chosen to yield best damping performance for the reference system that con- 


\begin{tabular}{|c|c|}
\hline \hline \multicolumn{2}{|c|}{ Hexagons } \\
\hline Circumscribed Radius $r$ & $0.0015 \mathrm{~m}$ \\
\hline Density (2D) $\rho$ & $215.77 \mathrm{kgm}^{-2}$ \\
\hline Coefficient of restitution $e$ & 0.50 \\
\hline Coefficient of friction $\mu$ & 0.50 \\
\hline \hline \multicolumn{2}{|c|}{ Triangles } \\
\hline Circumscribed Radius $r$ & $0.00086 \mathrm{~m}$ \\
\hline Density (2D) $\rho$ & $215.77 \mathrm{kgm}^{-2}$ \\
\hline Coefficient of restitution $e$ & 0.50 \\
\hline Coefficient of friction $\mu$ & 0.50 \\
\hline \hline
\end{tabular}

Table 1: Material properties of the particles for the simulations of fragmentation.

sist only of hexagonal grains (this corresponds to $L_{z}=0.1225 \mathrm{~m}$ ). Despite the simulated fragmentation, the height of the granular bed at rest remains fairly constant ( $\left.L_{0} \approx 0.039 \mathrm{~m}\right)$. Figure 3 ) shows snapshots of the system at rest for different degrees of fragmentation.

In Fig. 4 we show the FRFs for the five systems shown in Fig. 3. The FRF of the system is unaffected by the fragmentation of particles in the enclosure. This has important practical implications since the fragmentation during operation of a $\mathrm{PD}$ would not compromise the damping performance of the device, reducing the need for maintenance. This "universal" FRF observed in Fig. 4 is consistent with previous suggestions based on studies using different numbers of spherical grains (Sánchez et al., 2012). However, notice that the present work is taking due care of the shape that fragments have (they are different to the original particle) and still a universal FRF is found for the optimum $L_{z}$.

The universal response can be explained in terms of the effective "inelastic collapse" of the granular bed (Luding and McNamara, 1998; McNamara and Young, 1992). This phenomenon occurs when a granular sample that is being excited has a high density. Under such conditions, the granular pack achieves the dissipation of all the kinetic energy of the grains in a short time, even if the collisions between grains have a very high coefficient of restitution. In dense granular systems, the number of collisions per unit time grows quickly with the number of grains involved. Even a minute dissipation in each collision is enough to make the system, as a whole, fully dissipative. Since this condition of hav-

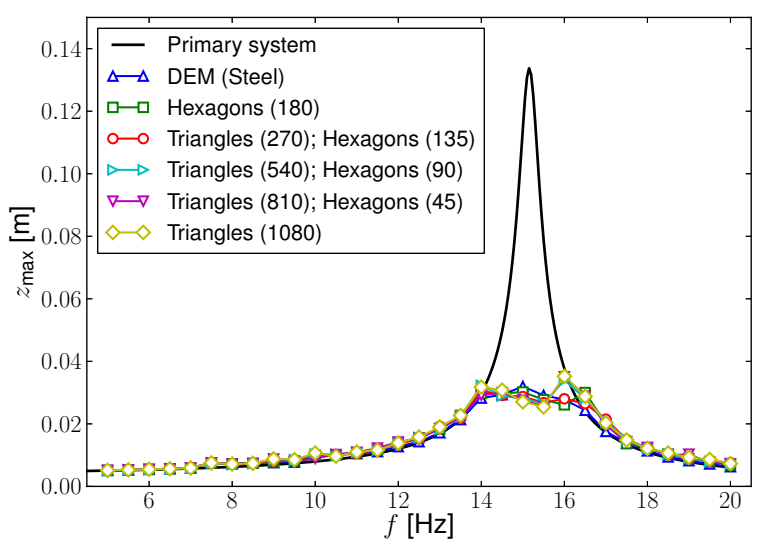

Figure 4: FRF for the system with the various distribution of particle size and shape shown in Fig. 3 . The total mass of the grain $m_{\mathrm{p}}=0.227 \mathrm{~kg}$ remains constant. Each curve corresponds to a different level of fragmentation of the hexagons (see legends). Blue triangles correspond to the FRF from 3D DEM simulations of the same primary system with an optimal PD with $N=250$ spherical particles and the same total mass but different interaction forces between the grains (Sánchez et al., 2012). The black line corresponds to the response of the system without the PD. 


\begin{tabular}{|c|c|}
\hline Property & Value \\
\hline \hline Young's modulus $E$ & $2.03 \times 10^{11} \mathrm{Nm}^{-2}$ \\
\hline Density & $8030 \mathrm{kgm}^{-3}$ \\
\hline Poisson's ratio $v$ & 0.28 \\
\hline Friction $\mu_{\mathrm{d}}$ & 0.3 \\
\hline Normal damping $\gamma_{\mathrm{n}}$ & $3.660 \times 10^{3} \mathrm{kgs}^{-1} \mathrm{~m}^{-1 / 2}$ \\
\hline Shear damping $\gamma_{\mathrm{s}}$ & $1.098 \times 10^{4} \mathrm{kgs}^{-1} \mathrm{~m}^{-1 / 2}$ \\
\hline Particle radius & $0.003 \mathrm{~m}$ \\
\hline \hline
\end{tabular}

Table 2: Material properties of the spheres for the 3D DEM simulations. For a description of the normal and shear damping coefficients involved in the particle-particle interaction force see (Sánchez et al., 2012).

ing a dense granular pack is always present for the motion of the granular bed at and around the resonant frequency for the optimum $L_{z}$, the response of the primary system is similar in all cases regardless of the material properties, shape of the grains and number of particles. This response is comparable to the response that the system would have if the entire set of grains were replaced by a single body with zero coefficient of restitution (Sánchez et al., 2012).

To further emphasize the universal character of the FRF, we include in Fig. 4 the results from a simulation of a three-dimensional (3D) PD with $N=250$ steel spheres (Sánchez et al., 2012). For these simulations, we have implemented a discrete element method (DEM) (Cundall and Strack, 1979) in $\mathrm{C}$. This code uses the model of Hertz-KuwabaraKono for normal interactions of the grains and the frictional model of Coulomb for the tangential interactions (Brilliantov et al., 1996; Pöschel and Schwager, 2004; Schäfer et al., 1996). The material properties that we have used for the spheres are presented in Table 2. As we can see, the curves from $2 \mathrm{D}$ and $3 \mathrm{D}$ simulations are similar to each other. This confirms that the universal FRF is also valid if dimensionality is changed.

\subsection{Effect of particles fusion}

PDs have become important as passive vibration control systems in harsh environments. In particular, they are used in environments with extreme temperatures (high and low) or with elevated pres- sures. Although the optimal PD has a universal response (independent of the material used for the grains and independent of the fragmentation of the particles), at high temperatures a wrong choice of materials may cause the welding of particles, which would reduce the effective number of grains in the cavity. It is known to reduce the number of collisions per unit time and prevent inelastic collapse.

In order to study the possible effect of the fusion of particles within the cavity, we have carried out simulations with square particles that are progressively replaced by bigger grains. In our simulations, we replace four square grains by a larger square with the area and mass equivalent to the total area and mass of the four grains removed. In this way, the total mass in the enclosure remains constant, but the number and size distribution of the particles change. We have used the optimal $L_{z}$ for the original system that consist in $N=128$ squares of radius $0.0021 \mathrm{~m}$ with $\mu=0.50$ and $e=0.50$. Figure 5 shows snapshots of the different systems simulated with varying degree of particle fusion.

In Fig. 6, we plot the FRFs for the different degrees of fusion shown in Fig. 5. The figure shows that for a large number of small square particles (see Fig. 5(a)) the response is optimum. This response coincides with the universal FRF also observed for hexagons in the previous section.

As the fusion of the grains progresses, the attenuation of the vibration degrades. In particular, a resonance peak starts to develop for frequencies below the natural frequency of the primary system (compare with the FRF of the system with an empty enclosure in Fig. 6). It is clear that the reduction of the total number of particles in the cavity compromises the ability of the PD to dissipate the kinetic energy. Therefore, appropriate maintenance tasks should be scheduled if working under conditions that may favor particle welding.

It is worth mentioning here that our observations indicate that whenever the granular layer in the PD exceeds four or five layers of grains, the effective inelastic collapse is achieved. This is consistent with previous studies on vibration of granular material (Chung et al., 2011), and PDs (Marhadi and Kinra, 2005; Sánchez et al., 2012). Therefore, the deterioration of the attenuation due to fusion only occurs when the number of granular layer decays below this threshold. 


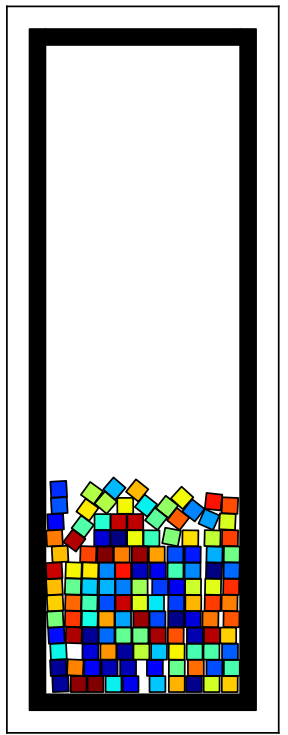

(a)

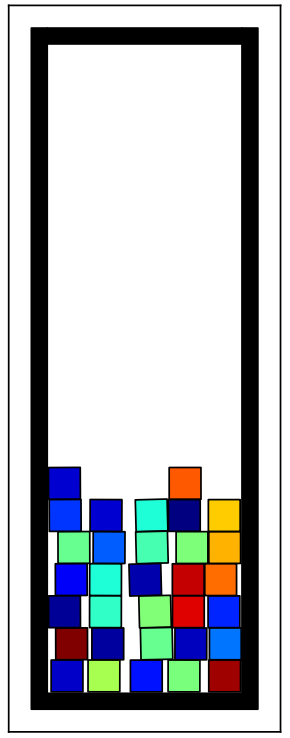

(b)

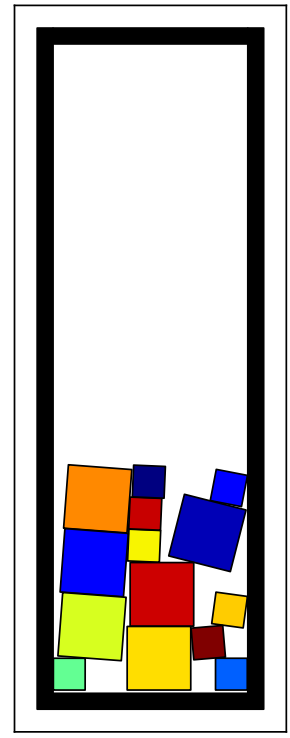

(c)

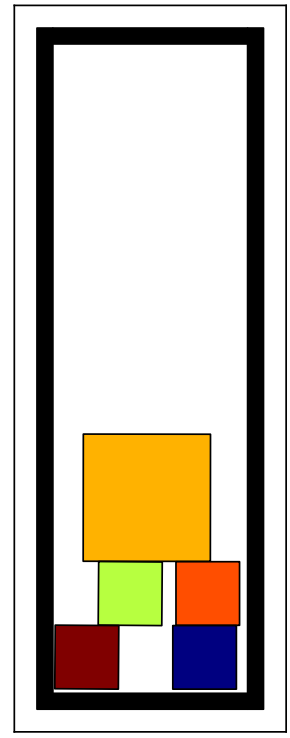

(d)

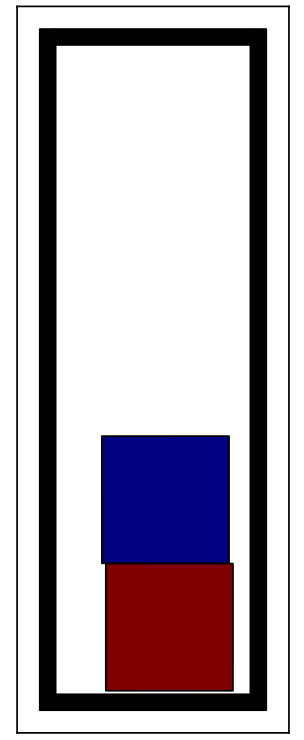

(e)

Figure 5: Snapshots of granular samples at rest. (a) 128 squares (circumscribed radius $r=0.0021 \mathrm{~m}$ ). (b) 32 squares resulting from welding sets of four squares from panel (a). (c) Six squares [from welding sets of 16 squares from (a)] mixed with eight squares [from welding sets of four squares from (a)]. (d) One square [welding 64 squares from (a)] mixed with four particles [welding sets of 16].(e) Two particles made out of 64 squares from (a) each. 


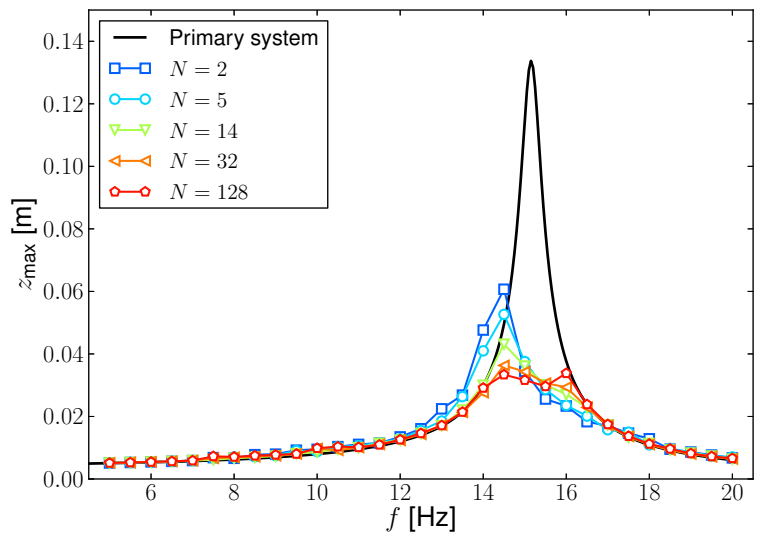

Figure 6: FRF for the system with the various distribution of sizes of square particles shown in Fig. 5 . The total mass of the grains $m_{\mathrm{p}}=0.227 \mathrm{~kg}$ remains constant. Each curve corresponds to a different level of fusion (see legends). The black full line corresponds to the response of the system without the PD.

\subsection{Effect of material properties and shape for non-optimal PDs}

From section 3.1, we conclude that the shape of the particles in the enclosure of a PD is not significant for the response of the system if the optimum $L_{z}$ is chosen. In this section we consider different grain shapes and coefficients of restitution to evaluate to what extent the shape of the particles and material properties can influence the behavior of the PD if the inelastic collapse is not achieved. In order to show the difference in the behavior when the inelastic collapse happens and when it does not, the number $N$ of particles was changed. The material density of the particles is adjusted when $N$ is changed so as to keep the total mass of the particles constant. Properties of the grain for these simulations are shown in Table 3.

Figure 7(a) shows the FRF for PDs with only two particles (where inelastic collapse is not likely) when different grain shapes and restitution coefficients are used. These results show, again, that the response of the PD is not longer universal if few particles are used. The FRF depends on the restitution coefficient, with a clear improvement in damping for smaller restitutions, as it should be expected. However, the different shapes of the grains in the cavity has very little impact on the FRF. Triangles, squares and hexagons yield similar results. The subtle differences between FRF for different particle shapes can be attributed to the small differences in the height of the granular bed at rest due to the different arrangements each grain shape can take.

When the number of layers of grains in the PD is somewhat larger, $N=64$, the response of the PD becomes independent of the coefficient of restitution (see Fig. 7(b)). This is in agreement with previous studies using spherical particles (Sánchez et al., 2012). The emergence of inelastic collapse makes the system to follow the universal FRF.

In Fig. 8, we show in more detail the maximum amplitude of vibration of the primary system at the resonance frequency $(f=14.5 \mathrm{~Hz})$ as a function of the coefficient of restitution for grains of different shapes. Once again, the response is independent of particle shape (whether there exist an inelastic collapse or not). If a large number of particles is used, the inelastic collapse also leads to a constant response as a function of restitution. However, if only a few particles are inserted in the enclosure, the attenuation is more effective as restitution is decreased.

\section{Conclusions}

We have considered the effect of fragmentation and fusion of particles in a PD on the vibration attenuation. The results of our simulations indicate that, if a sufficiently large number of particles are used (typically over six layers of grains), fragmentation of particles is unable to alter the response of the PD. In contrast, fusion will reduce the effective number of grains, preventing the inelastic collapse, which may eventually deteriorate the damping ability of the PD. Form a practical perspective, this implies that working under conditions where fragmentation is likely does not require maintenance of the PD (such as replacement of particles). However, if fusion is possible, regular maintenance inspections should be carried out. Notice however that fragmentation may eventually convert the granular sample into a fine powder. In such extreme cases a more careful study is necessary since the aero- 


\begin{tabular}{|c|c|c|c|}
\hline \multicolumn{2}{|c|}{ Big squares $N=2$} & \multicolumn{2}{|c|}{ Small squares $N=64$} \\
\hline Circumscribed radius $r$ & $0.017 \mathrm{~m}$ & Circumscribed radius $r$ & $0.0029 \mathrm{~m}$ \\
\hline Density $(2 \mathrm{D}) \rho$ & $197.08 \mathrm{kgm}^{-2}$ & Density $(2 \mathrm{D}) \rho$ & $201.10 \mathrm{kgm}^{-2}$ \\
\hline \multicolumn{2}{|c|}{ Big triangles $N=2$} & \multicolumn{2}{|c|}{ Small triangles $N=64$} \\
\hline Circumscribed & $0.019 \mathrm{~m}$ & Circumscribed radiu & $0.0034 \mathrm{~m}$ \\
\hline Density $(2 \mathrm{D}) \rho$ & $240.74 \mathrm{kgm}^{-2}$ & Density $(2 \mathrm{D}) \rho$ & $227.57 \mathrm{kgm}^{-2}$ \\
\hline \multicolumn{2}{|c|}{ Big hexagons $N=2$} & \multicolumn{2}{|c|}{ Small hexagons $N=64$} \\
\hline Circumscribed radius $r$ & $0.012 \mathrm{~m}$ & Circumscribed radius $r$ & $0.0024 \mathrm{~m}$ \\
\hline Density (2D) $\rho$ & $303.43 \mathrm{kgm}^{-}$ & Density (2D) $\rho$ & $237.05 \mathrm{kgm}^{-2}$ \\
\hline
\end{tabular}

Table 3: Material properties of the particles for simulations of Section 3.3.

dynamic interaction of the powder particles with the air in the cavity may significantly affect the response of the PD.

The results we have presented for PD containing grains of different shapes indicate that the geometry of the particles has not impact on the damping performance. Even if few particles are used - where the inelastic collapse is not at play and the FRF deviates from the universal response - a change in the particle shape does not affect significantly the response of the PD.

In summary, the use of a large number of particles in a PD ensures that a universal FRF will be obtained if the optimal enclosure height is used. This universality not only implies that the material properties of the grains are irrelevant (Sánchez et al., 2012), also the shape of the grains, and in particular their fragmentation, is unimportant. However, if few particles are inserted in the PD, the response will be sensitive to the material properties, whereas will remain insensitive to the particle shape.

\section{Acknowledgements}

LAP and CMC acknowledge support from CONICET (Argentina).

\section{References}

Araki, Y., I. Yokomichi, and J. Inoue (1985). Impact dampers with granular materials. Bulletin of JSME 28, 1466-1472.

Bai, X. M., L. M. Keer, Q. J. Wang, and R. Q. Snurr (2009). Investigation of particle damping mechanism via particle dynamics simulations. Granular Matter 11, 417-429.

Brilliantov, N. V., F. Spahn, J. M. Hertzsch, and T. Pöschel (1996). Model for collisions in granular gases. Physical Review E 53(5), 5382-5392.

Carlevaro, C. M. and L. A. Pugnaloni (2011). Steady state of tapped granular polygons. Journal of Statistical Mechanics: Theory and Experiment, P01007.

Catto, E. (2005). Iterative dynamics with temporal coherence. In Game Developer Conference, pp. $1-24$.

Catto, E. (2012). Box2D Physics Engine. Available from: http://www.box2d.org. [online; accessed Nov. 30, 2012].

Chen, T., K. Mao, X. Huang, and M. Y. Wang (2001, March). Dissipation mechanisms of nonobstructive particle damping using discrete element method. In Proceedings of SPIE, Smart Structures and Material: Damping and Isolation, Newport Beach, CA, pp. 294-301.

Chung, F. F., S. S. Liaw, and W. C. Chang (2011). Collective motion of inelastic particles between two oscillating walls. Granular Matter 13, 787794.

Cundall, P. A. and O. D. L. Strack (1979). A discrete numerical model for granular assemblies. Geotechnique 29, 47-65.

Duncan, M. R., C. R. Wassgren, and C. M. Krousgrill (2005). The damping performance of a single particle impact damper. Journal of Sound and Vibration 286(1-2), 123-144. 


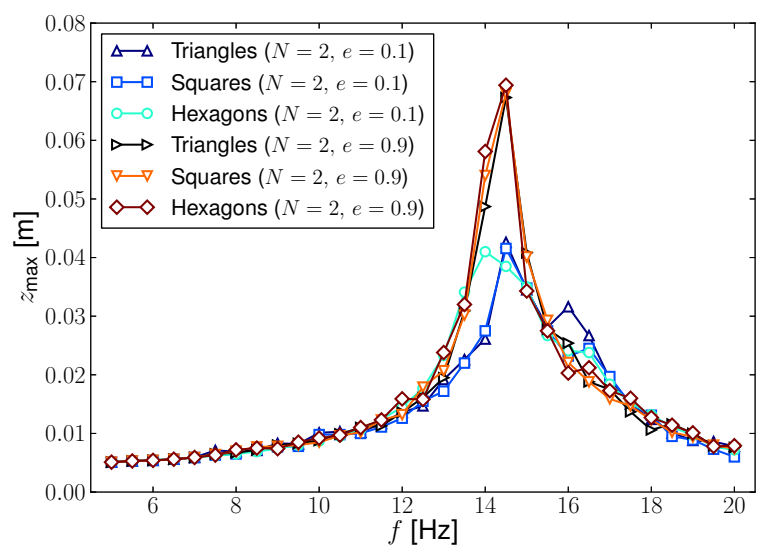

(a)

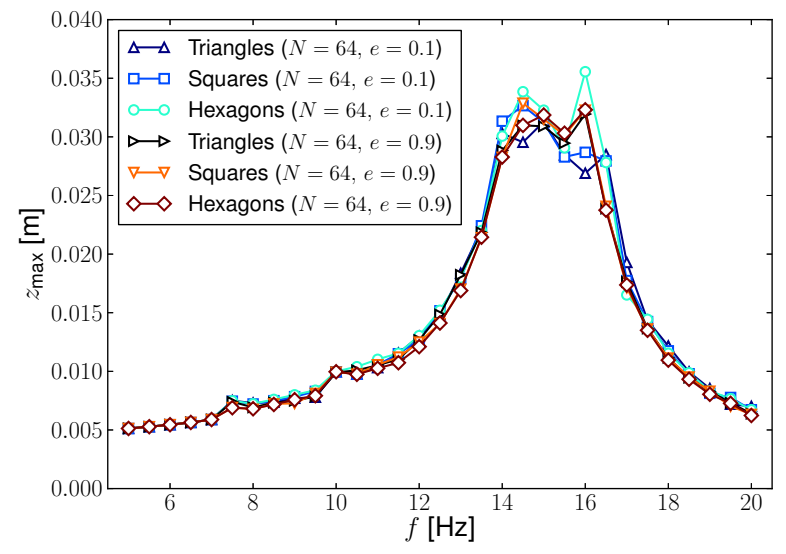

(b)

Figure 7: FRF for different particle shapes and restitution coefficients $e$. (a) Results for 2-particle systems containing triangles, squares or hexagons at two values of $e$. (b) Same as (a) for 64-particle systems.

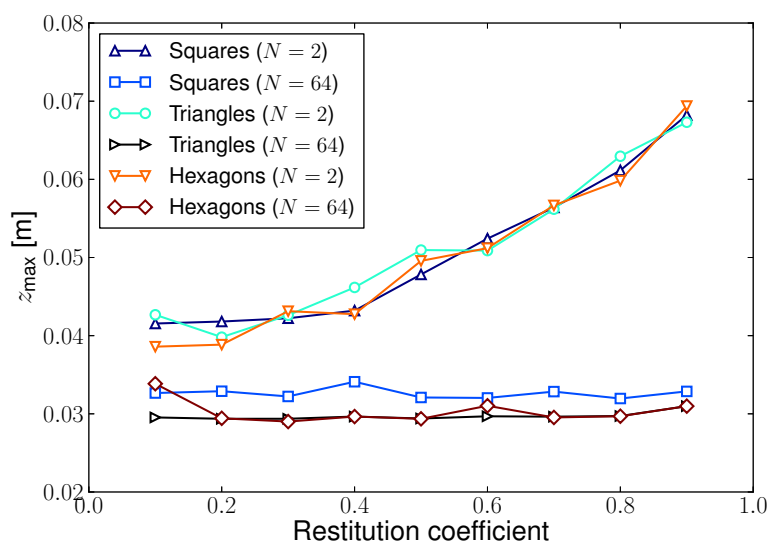

Figure 8: Amplitude of vibration $z_{\max }$ at $f=14.5$ $\mathrm{Hz}$ as a function of restitution coefficient for different number of particles and particle shape (see legend).

Ehrgott, R., H. V. Panossian, and G. Davis (2009). Modeling techniques for evaluating the effectiveness of particle damping in turbomachinery. In 50th AIAA/ASME/ASCE/AHS/ASC Structures, Structural Dynamics and Material Conference, Palm Springs.

Fang, X. and J. Tang (2006). Granular damping in forced vibration: qualitative and quantitative analyses. Journal of Vibration and Acoustic 128, 489-500.

Friend, R. D. and V. K. Kinra (2000). Particle impact damping. Journal of Sound and Vibration 233(1), 93-118.

Grubin, C. (1956). On the theory of the acceleration damper. Transactions of ASME. Journal of Applied Mechanics 23(3), 373-378.

Heckel, M., A. Sack, J. E. Kollmer, and T. Pöschel (2012). Granular dampers for the reduction of vibrations of an oscillatory saw. Physica A: Statistical Mechanics and its Applications 391(19), 4442-4447.

Liu, W., G. R. Tomlinson, and J. A. Rongong (2005). The dynamic characterisation of disk geometry particle dampers. Journal of Sound and Vibration 280, 849-861. 
Lu, Z., X. Lu, and S. F. Masri (2010). Studies of the performance of particle dampers under dynamic loads. Journal of Sound and Vibration 319, 5415-5433.

Luding, S. and S. McNamara (1998). How to handle the inelastic collapse of a dissipative hard-sphere gas with the TC model. Granular Matter 1(3), $113-128$.

Marhadi, K. S. and V. K. Kinra (2005). Particle impact damping: effect of mass ratio, material,and shape. Journal of Sound and Vibration 283, 433448.

Masri, S. F. (1970). General Motion of Impact Dampers. Journal Acoustical Society of America 47 (1B), 229-237.

McNamara, S. and W. R. Young (1992). Inelastic collapse and clumping in a onedimensional granular medium. Physics of Fluids 4(3), 496-504.

Panossian, H. V. (1992). Structural damping enhancement via non-obstructive particle damping technique. Journal of Vibration and Acoustics 114, 101-105.

Panossian, H. V. (2002). Non-obstructive particle damping experience and capabilities. In Proceedings of SPIE 4753, pp. 936-941.

Papalou, A. and S. F. Masri (1998). An experimental investigation of particle dampers under harmonic excitation. Journal of Vibration and Control 4, 361-379.

Pöschel, T. and T. Schwager (2004). Computational Granular Dynamics: Models and Algorithms. Berlin: Springer.

Ramachandran, S. and G. Lesieutre (2008). Dynamics and performance of a harmonically excited vertical impact damper. Journal of Vibration and Acoustics 130(2), 021008.

Saeki, M. (2002). Impact damping with granular materials in a horizontally vibrating system. Journal of Sound and Vibration 251 (1), 153-161.

Sánchez, M. and C. M. Carlevaro (2012). Nonlinear dynamic analysis of an optimal particle damper. Journal of Sound and Vibration (in press).
Sánchez, M. and L. A. Pugnaloni (2010). Modelling of a granular damper. Mecánica Computacional XXIX, 1849-1859.

Sánchez, M. and L. A. Pugnaloni (2011). Effective mass overshoot in single degree of freedom mechanical systems with a particle damper. Journal of Sound and Vibration 330(24), 5812-5819.

Sánchez, M., G. Rosenthal, and L. A. Pugnaloni (2012). Universal response of optimal granular damping devices. Journal of Sound and Vibration 331, 4389-4394.

Schäfer, J., S. Dippel, and D. E. Wolf (1996). Force schemes in simulations of granular materials. Journal de Physique I 6, 5-20.

Velichkovich, A. S. and S. V. Velichkovich (2001). Vibration-Impact Damper for Controlling the Dynamic Drillstring Conditions. Chemical and Petroleum Engineering 37(3-4), 213-215.

Xia, Z., X. Liu, and Y. Shan (2011). Application of particle damping for vibration attenuation in brake drum. International Journal of Vehicle Noise and Vibration 7(2), 178-194. 